\title{
Evaluación del Desempeño y Auténtica en el Modelo por Competencias en Secundaria: Un Estudio Mixto
}

\author{
Evaluation of Performance and Authentic in the Model by \\ Competences in Secondary: A Mixed Study
}

\author{
Aliosha Alderete Martínez ${ }^{1 *}$ \\ Katherina Edith Gallardo ${ }^{2}$ \\ ${ }^{1}$ Secretaria de Educación Pública, México \\ ${ }^{2}$ Instituto Tecnológico y de Estudios Superiores de Monterrey, México
}

\begin{abstract}
Este artículo presenta los resultados de un proceso de investigación educativa sobre la aplicación de algunos principios de evaluación del desempeño y auténtica en educación secundaria, en la asignatura de Historia, relacionando este tipo de evaluación con la satisfacción y el cambio educativo en el tema de evaluación de los estudiantes. El objetivo que persigue el estudio es comprender cómo indicen los procesos de evaluación en los estudiantes después de la aplicación de esos principios en el marco del Modelo Educativo Basado en Competencias, considerado uno de los cambios educativos más importantes de esta década en la mayoría de los sistemas educativos de los países latinoamericanos. Se realizó un estudio usando Métodos mixtos en una escuela pública. Un grupo de 35 estudiantes de segundo año participaron en el estudio. Se diseñaron tres instrumentos para la recopilación de datos: una rúbrica de rendimiento, una entrevista y un cuestionario. Los resultados obtenidos en términos de percepción del uso y la aplicación de la evaluación y el rendimiento auténticos permiten inferir que los estudiantes experimentaron satisfacción con estos nuevos procesos de evaluación y retroalimentación. También se analizó que las calificaciones de los estudiantes aumentaron, incluidos aquellos estudiantes con bajo rendimiento al comienzo del estudio. Las lecciones aprendidas a partir del estudio, así como las necesidades de investigación en este tema se discuten como parte de las reflexiones derivadas de la investigación.
\end{abstract}

Descriptores: Evaluación; Estudiante; Educación; Rendimiento escolar; Enseñanza.

This article presents results from an educational research process about the application of some principles of performance and authentic assessment in secondary level in the subject of History related to satisfaction and change of meaning about assessment of students. The objective follows the intention of understanding the impact of students after the application of those principles in the framework of Competency-based education model, considered one of the most important educational changes of this decade within most of the Latin-American countries in educational systems. A Mixed-method study was made in a public school. A group of 35 students from second year participated. Three instruments were developed for data collection purposes: A performance rubric, an interview as well as a questionnaire. The results achieved in terms of perception of the use and application of authentic evaluation and performance allow inferring that students experimented satisfaction with these new assessment process and feedback giving. It was also analyzed that students' grades reached higher level, including those students with low performance at the beginning of the study. Lessons learn and research needs in this topic are further discussed.

Keywords: Evaluation; Students; Education; Academic achievement; Teaching.

*Contacto: almaragon@gmail.com

ISSN: $1696-4713$

www.rinace.net/reice/

revistas.uam.es/reice
Recibido: $\quad 5$ de febrero 2018

$1^{\text {a }}$ Evaluación: 23 de abril 2018

$2^{\text {a }}$ Evaluación: 19 de mayo 2018

Aceptado: $\quad 8$ de junio 2018 


\section{Introducción}

Para un número importante de países de América Latina, la evaluación del aprendizaje se ha convertido en los últimos años en tema de urgente atención en las agendas educativas (Murillo y Román, 2008). Por ejemplo, en el marco del proyecto Metas 2021 de Iberoamérica, el tema de evaluación del aprendizaje se encuentra como prioritario en la lista de mejoras a realizar en los sistemas educativos (OEI, 2010). El logro del cambio y la mejora de las prácticas en este rubro sigue siendo un anhelo por cumplir en diferentes naciones (Gallardo, 2013; Hidalgo y Murillo, 2017). A partir de estas aseveraciones, se infiere que el proceso de cambio educativo, que hace más de una década impulsó a muchos países latinoamericanos a reformar sus modelos hacia el trabajo por competencias y sus sistemas de evaluación en función del nuevo modelo (como lo fue el caso de México en el año 2008), sigue su curso buscando consolidarse.

Este estudio trata justamente de la puesta en marcha de una iniciativa para fortalecer el proceso de cambio en el tema de evaluación de desempeño y auténtica, que es la forma en que se define y denomina la evaluación en el Modelo Educativo Basado en Competencias (MEBC). Se sabe que el tema de evaluación de desempeño y auténtica en educación básica es reconocido como un eje fundamental para consolidar el trabajo educativo bajo el MEBC (Argudín, 2010; Monereo, 2009). No obstante, podría afirmarse que este fenómeno educativo, en la actualidad, es escasamente investigado, a pesar de ser parte de un modelo que se ha puesto en marcha en varios países latinoamericanos (Ferrer, 2006; Wolfe, Valverde y Esquivel, 2000).

En los últimos 10 años, según lo reportado en sitios especializados en investigación, se ha publicado en español un artículo de investigación (López, 2011) que aborda el tema del MEBC en educación básica, mientras que el número de artículos publicados en inglés sobre este tema asciende a 115, rebasando por mucho la producción científica en español (consultas hechas en Google Académico y Proquest Eric, respectivamente). Sin embargo, de estos 115 artículos, solo 9 se enmarcan en la educación secundaria y evaluación del desempeño y auténtica.

Estos datos sobre la producción científica en el tema permiten inferir lo urgente que es llevar a cabo procesos de indagación para entender cómo se suscita el cambio educativo desde el trabajo con el modelo por competencias, dado que ya han pasado algunos años desde su inserción (Flores-Kastanis y De la Torre, 2010). Así, es prioritario que, en la agenda de la investigación en Latinoamérica, se contemplen estudios que permitan entender cómo se presenta en las aulas la transformación de ciertas prácticas alrededor del trabajo con el MEBC. En consonancia con esta necesidad, este trabajo de investigación es un esfuerzo por comprender la transformación esperada en el campo de la evaluación educativa, desde el modelo ejercido en el nivel educativo de secundaria.

\section{Revisión de la literatura}

\subsection{Cambio educativo a partir de la inserción del modelo por competencia}

Ante el avance económico a partir de fenómenos como la internacionalización y la globalización; el crecimiento del mercado y la competitividad han ganado terreno en diferentes naciones (Ginés, 2004; Hopenhayn, 2003). Los sectores productivos exigen que los cambios educativos que podrían acercar a los ciudadanos al progreso de sus naciones 
dejen de ser una promesa, para convertirse en resultados. Paralelamente, organismos internacionales como el Banco Mundial (BM), OCDE, UNESCO y UNICEF (De Ketele, 2008, p. 7), han expresado preocupación por el pobre rendimiento por parte de los sistemas educativos, exigiendo que los procesos de rendición de cuenta de los avances en materia de desarrollo de capacidades no se puedan expresar solo a través de calificaciones, sino a través del aseguramiento del nivel de capacidades que logran los estudiantes a lo largo de su educación.

Así, la educación por competencias emerge con fuerza a mediados de 80's. Según la UNESCO, la educación basada en competencias toma en cuenta tanto aspectos contextuales como resultados de aprendizaje. Además, cuenta con criterios de ejecución o desempeño que implica no sólo asegurar el saber hacer, sino también otros saberes, como el convivir y el ser, lo cual genera un sentido de mayor integración de elementos tan importantes como los conocimientos en el actuar de los seres humanos (Aguerrondo, 2008). Algunos autores aseguran que la germinación del modelo por competencias es uno de los avances más importante de nuestros tiempos, se identifica con el desempeño eficaz y eficiente de un papel o función, reconocido y aprobado por una comunidad científica y aceptado como modelo para encontrar soluciones y crear desarrollo (Chagoyan, 2013, De la Orden Hoz, 2011; Tobón, 2008). Este modelo ha traído problemas a los docentes para dominar el enfoque, pero por otro lado el alumno puede llegar a tener otra manera de aprender en el proceso enseñanza-aprendizaje, habilidad que puede impactar en la solución de problemas de la vida real (Chong, 2013).

Sin embargo, lo que no se ha podido resolver a ciencia cierta es en qué etapa del cambio educativo se encuentra la inserción del modelo educativo por competencias si se intenta ubicar su penetración en los sistemas educativos. Es decir, si se ve en contraste con el proceso de cambio (Fullan, 2002) sería difícil determinar si se encuentra en alguna de estas fases: (1) iniciación, (2) implementación o (3) institucionalización. Aunque parecería que ya se encuentra en la etapa de institucionalización por el número de años transcurridos desde su inserción, la realidad es que se cuenta con escasa investigación sobre sus primeros frutos desde su incorporación en la educación básica (primaria y secundaria) aplicándose en el 2008 en el caso del sistema educativo mexicano. Por lo anteriormente expuesto, se juzga prioritario contar con mayor investigación sobre el trabajo relacionado con su planeación, puesta en marcha y evaluación.

\subsection{Evaluación de desempeño y auténtica}

Uno de los puntos de mayor controversia al hablar de la inserción del modelo educativo basado en competencias es la forma en que se lleva a cabo la evaluación, la cual se denomina evaluación del desempeño y auténtica (Hancock, 2007; Palm, 2008).

La evaluación auténtica, es un tipo de evaluación de enfoque naturalista que busca reflejar prácticas y desempeños que realmente están ocurriendo ante un reto de aprendizaje, los procesos de evaluación van más allá de emitir una única y correcta solución (Gallardo, 2013). Este tipo de evaluación aún es joven en el ámbito de educación básica (primaria y secundaria), en comparación con los años que lleva aplicándose en el ámbito de formación profesional. Sin embargo, es posible y deseable insertar las prácticas de evaluación del desempeño y auténtica para ir en consonancia con el ejercicio del modelo educativo (Monereo, 2009, Rodríguez y Gallardo, 2017; Tobón, 2008). 
La evaluación del desempeño requiere de observación, seguimiento y medición de conductas de los alumnos en el momento que se encuentren ejecutando alguna acción relacionada con el proceso de aprendizaje, ya sea de manera individual o colaborativa. Por tal razón, se deben fundamentar las decisiones que se tomen alrededor de la planeación, instrumentación, interpretación de resultados y procesos de mejora en marcos teóricos robustos (Escamilla et al., 2016).

Este dinamismo ha impactado diversas formas de procesos de evaluación que se están promoviendo en educación superior. Sin embargo, el tratar de implementar estas evaluaciones en educación secundaria, representa un reto para directivos y profesores, dada la distancia que existe entre las prácticas de evaluación que exige el modelo y a que las evaluaciones no corresponden "tradicionalmente" a las formas de evaluación que se llevan a cabo en los distintos niveles educativos. Es preciso recordar que, para que estos cambios se susciten, deben darse "desde el punto de vista del profesor individual, el estudiante, los padres y la administración si queremos entender las acciones y las reacciones de cada uno" (Fullan, 2002, p. 6).

Adicionalmente, es preciso indicar que la evaluación auténtica agrupa todo un conjunto de alternativas frente a la evaluación tradicional. Así, el alumno mejora o crea un producto o responde con soluciones a una necesidad. Este proceso permite evaluar cómo realiza el proceso de transferencia de sus conocimientos, plasmándolos en resultados observables (Bravo y Fernández, 2000). Aunque se entiende valiosa y necesaria, generalmente, el problema de aplicar este tipo de evaluación estriba en realizarla con grupos numerosos, sumado al poco dominio conceptual y técnico del proceso, lo cual puede derivar en caer en subjetividades al momento de valorar el desempeño de los estudiantes.

A nivel secundaria, es prácticamente desconocida la evaluación auténtica y es poco promocionada su utilidad. Sin embargo, si aspiramos a que nuestros estudiantes desarrollen las competencias necesarias para la vida, como se indica en el perfil de egreso, se deben modificar las prácticas educativas que puedan mejorar el proceso de aprendizaje, y en este caso en específico, es preciso realizar cambios en los procesos de evaluación y retroalimentación (Gallardo, 2013).

\subsection{Enseñanza y evaluación en la disciplina de historia en secundaria}

La enseñanza de la historia en la educación secundaria presenta situaciones particulares que atender, dado que el profesor debe enseñar, de acuerdo con los planes y programas educativos, con su propia ideología respecto a temas históricos, aunado a las nociones previas que tiene el estudiante sobre lo que es la disciplina (Carretero, 2000). Lo cierto es que los alumnos nunca llegan con la mente en blanco a tomar esta materia, la estudiaron en la educación primaria, aunque de manera superficial. En secundaria, es un reto forjar en la mente de los estudiantes cambios en la comprensión y detalles de ciertos eventos históricos, teniendo como objetivo la ampliación de las ideas para comprender racionalmente los cambios del mundo y, con base en esto, se puedan desenvolver en él. Por lo anterior, la motivación relacionada con el estudio de la historia debe ser tratada con suma atención al momento de aplicar prácticas pedagógicas que promuevan el cambio de conceptos en la mente de los estudiantes, que aprendan a ver la historia como una materia que les permitirá cambiar su conocimiento, es fundamental para lograr aprendizajes significativos (Carretero, 2000; Casal, 2011). 
Lo cierto es que aprender historia, acompañado de buenas prácticas pedagógicas, ayuda enormemente en la formación de estudiantes de educación secundaria. Asimismo, coadyuva a disipar ideas arraigadas, fundamentadas o no, de que la materia de historia es monótona y aburrida (Casal, 2011); un análisis de los modelos didácticos y los procesos de evaluación puede ser de gran utilidad para realizar los cambios educativos pertinentes, que permitan ver a la historia como parte de un todo y que lleve a los estudiantes a entender que su nación es parte del mundo. "La historia no es un mero saber de anticuario, contiene una manera de concebir qué somos y cómo debemos actuar. Su sustancia es el tiempo, el devenir, el cambio, no el acontecimiento" (Casal, 2011, p. 79)

En cuanto a las regulaciones sobre el aprendizaje de historia emitidas en México por la Secretaría de Educación Pública de México (SEP), bajo el mebc, se puede afirmar que es un área de estudio que debe propiciar el desarrollo de tres competencias principalmente: (1) comprensión del tiempo y el espacio históricos; (2) manejo de información histórica; y (3) formación de una conciencia histórica para la convivencia (SEP, 2011). Se parte de la consigna de crear un programa de estudio congruente con los rasgos de perfil de egreso de educación básica, para lo cual se llevaron a cabo una serie de reformas curriculares: el 384 para planes y programas de educación secundaria y publicado el 26 de mayo de 2006 en el Diario Oficial de la Federación (SEP, 2013), a partir de esto se identificaron una serie de competencias relevantes para que puedan lograr una vida plena y productiva.

A continuación, se presenta el cuadro 1 con la información relacionada con las competencias y subcompetencias relacionadas con el estudio de Historia. En aras de conocer, desde la percepción de los estudiantes, cómo la evaluación de desempeño y auténtica en la disciplina de Historia favorece el desarrollo de las competencias declaradas, se consideró pertinente realizar el proceso de indagación que se presenta en este artículo.

El punto de partida es el interés de los investigadores, dada la escasa producción científica al respecto. Un segundo interés, igualmente importante, es inferir en qué etapa se encuentra el proceso de cambio educativo derivado del trabajo con el MEBC, independientemente de que todo apunta a que se encuentra en la etapa de institucionalización.

Cuadro 1. Competencias del programa de Historia para educación media básica

Comprensión del tiempo y espacio histórico

Desarrollo de las nociones de tiempo y de espacio históricos.

Desarrollo de habilidades para comprender, comparar y analizar hechos.

Reconocer el legado del pasado y contar con una actitud crítica sobre el presente y devenir de la humanidad.

Manejo de información histórica

Expresar puntos de vista fundamentados sobre el pasado.

Movilizar conocimientos, habilidades y actitudes para seleccionar, analizar y evaluar críticamente fuentes de información.

Formación de una conciencia histórica para la convivencia.

Desarrollan conocimientos, habilidades y actitudes para comprender cómo las acciones, los valores y las decisiones del pasado impactan en el presente y futuro de las sociedades y de la naturaleza.

Fomentan el aprecio por la diversidad del legado cultural, además del reconocimiento de los lazos que permiten a los alumnos sentirse parte de su comunidad, de su país y del mundo.

Fuente: Elaborado a partir de SEP (2011). 


\section{Método}

El diseño del estudio es descriptivo abordado desde Métodos mixtos, para el cual se optó por un modelo concurrente, donde el aspecto cualitativo tuvo mayor importancia para comprender, desde la perspectiva del alumno, la vivencia del proceso de evaluación del desempeño y auténtica (Creswell y Plano, 2007; Valenzuela González y Flores-Kastanis, 2013). Cabe destacar que los datos cualitativos se trataron siguiendo el enfoque de análisis fenomenológico (Tójar, 2006). Se consideró pertinente que este diseño metodológico dado que, a la fecha, no existe suficiente literatura enfocada en la forma que deben adoptarse las nuevas prácticas evaluativas desde el enfoque de competencias en educación básica.

Esta investigación se realizó en una secundaria pública del municipio de Texcoco en el Estado de México, México, en la cual se estudia la materia de Historia bajo las normas establecidas por la Nueva Reforma Educativa (SEP, 2011). La escuela se encuentra en una zona suburbana de 235,151 habitantes, de acuerdo con censo de 2010. Se atiende a un total de 30 grupos (10 por grado) con aproximadamente 1000 estudiantes de secundaria en dos turnos. En el estudio participaron 35 estudiantes de segundo de secundaria, 20 hombres y 15 mujeres. Sus edades oscilan entre 13 y 15 años.

Se elaboraron tres instrumentos:

- Rúbrica de desempeño: se elaboró una rúbrica de desempeño para conducir el proceso de emisión de reportes y retroalimentación respectiva. Esta rúbrica se hizo expresamente para evaluar el desempeño de los estudiantes en el desarrollo de una tarea integradora relacionada con el tema de Derechos Humanos y su evolución en el tiempo para trabajar de manera transversal, a los temas marcados en el programa. Así, se determinaron una serie de actividades que, en su conjunto, conformaron el desarrollo del proyecto con base en las competencias y subcompetencias declaradas por la instancia reguladora del proceso educativo (cuadro 2).

- Entrevista semiestructurada: se diseñó una entrevista para recoger las percepciones de los estudiantes una vez culminado el proceso de evaluación (ver Apéndice A).

- Encuesta: sobre la evaluación auténtica y del desempeño, se aplicó a 33 estudiantes de los 35 en lista. Arrojó un índice de consistencia interna a través del cálculo de Alpha de Cronbach de 0.76, el cual es un valor aceptable que permite inferir sobre la suficiencia de la consistencia interna del instrumento. Estuvo conformada por 18 preguntas enfocadas en averiguar: (a) prácticas del profesor respecto a la evaluación (pregunta 1 a la 5); (b) aprendizajes esperados a partir de los resultados de evaluación (6 a la 9); (c) percepción sobre la evaluación de desempeño y auténtica (10 a la 15); y (d) percepción sobre preferencias como estudiante ante prácticas de evaluación (16 a 18) (ver Apéndice B). 
Cuadro 2. Rúbrica de desempeño relacionada con las competencias a evaluar en el proyecto relacionado con Historia

\begin{tabular}{|c|c|c|c|c|}
\hline \multicolumn{2}{|c|}{ COMPETENCIAS A EVALUAR } & RECUPERACIÓN & COMPRENSIÓN & ANÁLISIS \\
\hline & Subcompetencias & Nivel 1 Recuperación & Nivel a Comprensión & Nivel 3 Análisis \\
\hline \multirow{3}{*}{$\begin{array}{l}\text { 1. Comprensión del } \\
\text { tiempo y espacio } \\
\text { histórico }\end{array}$} & $\begin{array}{l}\text { 1.1 Desarrollo de las } \\
\text { nociones de tiempo y de } \\
\text { espacio históricos. }\end{array}$ & $\begin{array}{l}\text { Identifica los momentos históricos } \\
\text { relacionados con derechos en } \\
\text { diferentes sucesos mundiales del siglo } \\
\text { XIX al siglo XXI (en diferentes países } \\
\text { como EE.UU., Francia, México, etc.) } \\
\text { conjuntados en una línea de tiempo. }\end{array}$ & $\begin{array}{l}\text { Explica la relación de los momentos } \\
\text { históricos plasmados en la línea de } \\
\text { tiempo con el establecimiento de } \\
\text { derechos de las personas en México } \\
\text { y diferentes países, entre el siglo } \\
\text { XIX y el siglo XXI. }\end{array}$ & $\begin{array}{l}\text { Asocia diferentes eventos históricos } \\
\text { relacionados con los derechos humanos } \\
\text { y emite ideas sobre sus características o } \\
\text { elementos comunes (en cuáles } \\
\text { sociedades o países lucharon por la } \\
\text { libertad, la igualdad, etc.) }\end{array}$ \\
\hline & $\begin{array}{l}\text { 1.2 Desarrollo de } \\
\text { habilidades para } \\
\text { comprender, comparar y } \\
\text { analizar hechos }\end{array}$ & $\begin{array}{l}\text { Describe la vida cotidiana de las } \\
\text { sociedades en los periodos de tiempo } \\
\text { que se hicieron algunas de las } \\
\text { proclamaciones de derechos } \\
\text { ciudadanos más importantes en la } \\
\text { historia }\end{array}$ & $\begin{array}{l}\text { Explica las relaciones sociales que } \\
\text { existían en las sociedades en los } \\
\text { periodos de tiempo que se hicieron } \\
\text { declaraciones importantes de } \\
\text { derechos ciudadanos. }\end{array}$ & $\begin{array}{l}\text { Relaciona cómo las formas de vida de } \\
\text { una sociedad van dando las pautas para } \\
\text { la consecución de algunos derechos } \\
\text { ciudadanos, cómo se integran las } \\
\text { formas de vida con las declaraciones de } \\
\text { derechos. }\end{array}$ \\
\hline & $\begin{array}{l}\text { 1.3. Reconocer el legado } \\
\text { del pasado y contar con } \\
\text { una actitud crítica sobre } \\
\text { el presente y devenir de } \\
\text { la humanidad. }\end{array}$ & $\begin{array}{l}\text { Nombran situaciones históricas que } \\
\text { hicieron posible que se dieran } \\
\text { reformas legales para proteger } \\
\text { derechos o reforzar derechos de las } \\
\text { personas. }\end{array}$ & $\begin{array}{l}\text { Explican por qué después de un } \\
\text { conflicto armado, las sociedades } \\
\text { procuran reforzar sus derechos y } \\
\text { libertades para que dichas } \\
\text { situaciones no se vuelvan a repetir. }\end{array}$ & $\begin{array}{l}\text { Argumenta las razones de las } \\
\text { sociedades que han sufrido conflictos } \\
\text { armados y su necesidad de protección } \\
\text { jurídica por medio de la declaración de } \\
\text { derechos para que dichas situaciones no } \\
\text { se vuelvan a presentar sin que tengan } \\
\text { una protección. }\end{array}$ \\
\hline \multirow[t]{2}{*}{$\begin{array}{l}\text { 2. Manejo de } \\
\text { información } \\
\text { histórica }\end{array}$} & $\begin{array}{l}\text { 2.1 Expresar puntos de } \\
\text { vista fundamentados } \\
\text { sobre el pasado. }\end{array}$ & $\begin{array}{l}\text { Describen de manera sencilla pero } \\
\text { correcta que representan los } \\
\text { materiales elegidos, porqué eran así } \\
\text { antes, cómo es que fueron cambiando } \\
\text { a través del tiempo y cómo esos } \\
\text { cambios se relacionan con los } \\
\text { derechos de las personas }\end{array}$ & $\begin{array}{l}\text { Explican de manera sencilla pero } \\
\text { adecuada cuáles eran los materiales } \\
\text { de las cosas, por qué eran así, cómo } \\
\text { fueron cambiando y cuál es la } \\
\text { relación entre el cambio de las cosas } \\
\text { y los derechos de las personas. }\end{array}$ & $\begin{array}{l}\text { Relacionan cómo los materiales de las } \\
\text { cosas han ido cambiando con el paso del } \\
\text { tiempo adecuándose también a los } \\
\text { derechos de las personas }\end{array}$ \\
\hline & $\begin{array}{l}\text { 2.2 Movilizar } \\
\text { conocimientos, para } \\
\text { seleccionar, analizar y } \\
\text { evaluar críticamente. }\end{array}$ & $\begin{array}{l}\text { Describir los materiales presentados y } \\
\text { su relación histórica con los derechos } \\
\text { de las personas }\end{array}$ & $\begin{array}{l}\text { Representa con los materiales } \\
\text { presentados cómo era el uso de los } \\
\text { mismos y cómo estos materiales han } \\
\text { ido cambiando con el tiempo. }\end{array}$ & $\begin{array}{l}\text { Justifica el uso de los materiales en su } \\
\text { tiempo y el por qué éstos tuvieron que } \\
\text { cambiar para adecuarse a los derechos } \\
\text { de las personas. }\end{array}$ \\
\hline
\end{tabular}




\begin{tabular}{|c|c|c|c|c|}
\hline \multirow{3}{*}{$\begin{array}{l}\text { 3. Formación de } \\
\text { una conciencia } \\
\text { histórica para la } \\
\text { convivencia. }\end{array}$} & \multirow[t]{2}{*}{$\begin{array}{l}3.1 \text { Desarrollan } \\
\text { conocimientos, } \\
\text { habilidades y actitudes } \\
\text { para comprender cómo } \\
\text { las acciones, los valores y } \\
\text { las decisiones del pasado } \\
\text { impactan en el presente y } \\
\text { futuro de las sociedades y } \\
\text { de la naturaleza. }\end{array}$} & $\begin{array}{l}\text { Describir cómo era la vida de las } \\
\text { personas entrevistadas, de acuerdo a } \\
\text { la información obtenida y de las } \\
\text { observaciones en la entrevista. Listar } \\
\text { diferencias y similitudes en los } \\
\text { derechos que tenían los entrevistados } \\
\text { y los que ahora tienen ellos. Describir } \\
\text { cómo era la vida de las personas } \\
\text { entrevistadas. De acuerdo a la } \\
\text { información obtenida y de las } \\
\text { observaciones en la entrevista, listar } \\
\text { diferencias y similitudes en los } \\
\text { derechos que tenían los entrevistados } \\
\text { y los que ahora tienen ellos }\end{array}$ & $\begin{array}{l}\text { Explican cómo era la vida de los } \\
\text { entrevistados cuando tenían su edad } \\
\text { y cómo es que se fueron dando las } \\
\text { diferencias que hay entre los } \\
\text { derechos que ellos tenían y los que } \\
\text { hay ahora para los jóvenes }\end{array}$ & $\begin{array}{l}\text { Argumenta cómo se fueron dando los } \\
\text { cambios en los derechos de las personas } \\
\text { con el paso del tiempo, y puede dar una } \\
\text { opinión respecto de las diferencias que } \\
\text { existen hoy con los derechos de antes. }\end{array}$ \\
\hline & & $\begin{array}{l}\text { Reconocen que existen los Derechos } \\
\text { Humanos, han oído de ellos, pero no } \\
\text { los relacionan con su vida ni saben la } \\
\text { importancia que tienen, algunos } \\
\text { expresan uno o varios derechos } \\
\text { humanos sin poder relacionarlos con } \\
\text { su persona. * }\end{array}$ & $\begin{array}{l}\text { Explican uno o varios derechos, } \\
\text { entienden que para detener una } \\
\text { violación a derechos de los niños no } \\
\text { deben ser pasivos ante su presencia } \\
\text { y que los derechos de los niños son } \\
\text { parte inherente de su persona. }\end{array}$ & $\begin{array}{l}\text { Relacionan los derechos humanos no } \\
\text { sólo con su persona sino con una } \\
\text { comunidad. Saben que el bullying es una } \\
\text { agresión a los derechos humanos y } \\
\text { pueden tomar acciones que les permitan } \\
\text { detener esta situación }\end{array}$ \\
\hline & $\begin{array}{l}\text { 3.2 Fomenta el aprecio } \\
\text { por la diversidad del } \\
\text { legado cultural, además } \\
\text { del reconocimiento de los } \\
\text { lazos que permiten a los } \\
\text { alumnos sentirse parte de } \\
\text { su comunidad, de su país } \\
\text { y del mundo. }\end{array}$ & $\begin{array}{l}\text { Describen ante sus compañeros uno } \\
\text { de los derechos humanos elegidos, } \\
\text { pueden hablar algo acerca de su } \\
\text { historia y desarrollo hasta nuestros } \\
\text { días y pueden describir también como } \\
\text { se afectan estos derechos en su } \\
\text { comunidad }\end{array}$ & $\begin{array}{l}\text { Entienden la importancia de los } \\
\text { derechos humanos al hacer su } \\
\text { presentación, pueden explicar ante } \\
\text { sus compañeros y otros docentes } \\
\text { porque es importante su divulgación } \\
\text { y defensa, se interesan en participar } \\
\text { en su comunidad y círculos } \\
\text { cercanos. }\end{array}$ & $\begin{array}{l}\text { Además de reconocerse como parte } \\
\text { importante de su comunidad, mantienen } \\
\text { el interés por participar socialmente } \\
\text { para hacer saber a niños y personas de } \\
\text { sus círculos cercanos la importancia de } \\
\text { los derechos humanos y su defensa y } \\
\text { promoción para una convivencia sana y } \\
\text { pacífica. }\end{array}$ \\
\hline
\end{tabular}

derechos de los niños, participan activamente como defensores o integrantes de colectivos en campañas escolares para prevenir y erradicar el bullying. 
Para fines de contrastación, se tomaron como datos las calificaciones obtenidas por los 35 estudiantes a lo largo del proceso de evaluación sobre esta tarea integradora.

Procedimiento: se hizo del conocimiento de los estudiantes la inserción de prácticas de evaluación de desempeño a partir del cuarto y quinto bloque de la materia de Historia. Esta explicación se acompaña con ideas sobre la importancia de saber buscar información, la responsabilidad del trabajo individual y en equipo, así como la importancia de las tareas en torno al proyecto sobre Derechos Humanos. Posteriormente, se realizaron los procesos parciales de retroalimentación a las actividades y finalmente al proceso de desarrollo del proyecto de forma global. Se solicitó a los estudiantes de manera voluntaria sostener una entrevista sobre el proceso de evaluación. Se entrevistaron a seis estudiantes con el debido consentimiento de la escuela. Las entrevistas se grabaron, se transcribieron y se analizaron con ayuda del programa Atlas.ti. Se cuidaron los datos personales, por lo que se codificaron las afirmaciones por alumno. En cuanto a los reportes de retroalimentación sobre la evaluación de desempeño, se hicieron con apoyo del sistema COMPETERE (software de uso interno del Tecnológico de Monterrey, creado con fondos del proyecto Novus 2014, 2015, 2016).

\section{Resultados}

Este proyecto permitió apreciar la percepción de los estudiantes acerca de su proceso de aprendizaje y rendimiento académico a través de la evaluación de desempeño y auténtica, a partir de indagaciones de tipo cualitativo y cuantitativo. En ese orden se presentan los resultados.

\subsection{Resultados de tipo cualitativo}

Se realizaron seis entrevistas a participantes en el estudio, lo cual permitió comprender la percepción de seis estudiantes. Del proceso emergieron 2 familias con diversas categorías y códigos. Las figuras 1 y 2 ilustran las familias conformadas.

En la Familia $1[\mathrm{~F} 1]$ se formó alrededor de percepción de los estudiantes sobre el proceso de evaluación. Se les preguntó si sabían lo que era una competencia. Se apreció que en general existe confusión respecto al concepto, hubo razonamientos como:

No necesariamente tienes que ser el mejor o que esté bien, sino que lo que sea tu creas que está bien. (A22)

Mide la capacidad de alguien en base a algunos proyectos o trabajos que pongan. (A 17)

Es ver lo que sabes o lo que puedes aprender con educación y desempeño. (A5)

Al preguntarles acerca de su propia interpretación sobre lo que es la evaluación del desempeño y auténtica, se apreció que sus respuestas fueron más consistentes:

Es un sistema que nos muestra cómo vamos avanzando a través de proyectos. (A 17)

Los trabajos que hacíamos era lo que aprendimos en el año y lo demostramos con los trabajos. (A5)

La evaluación auténtica es lo que vamos trabajando, lo que hacemos. (A23) 


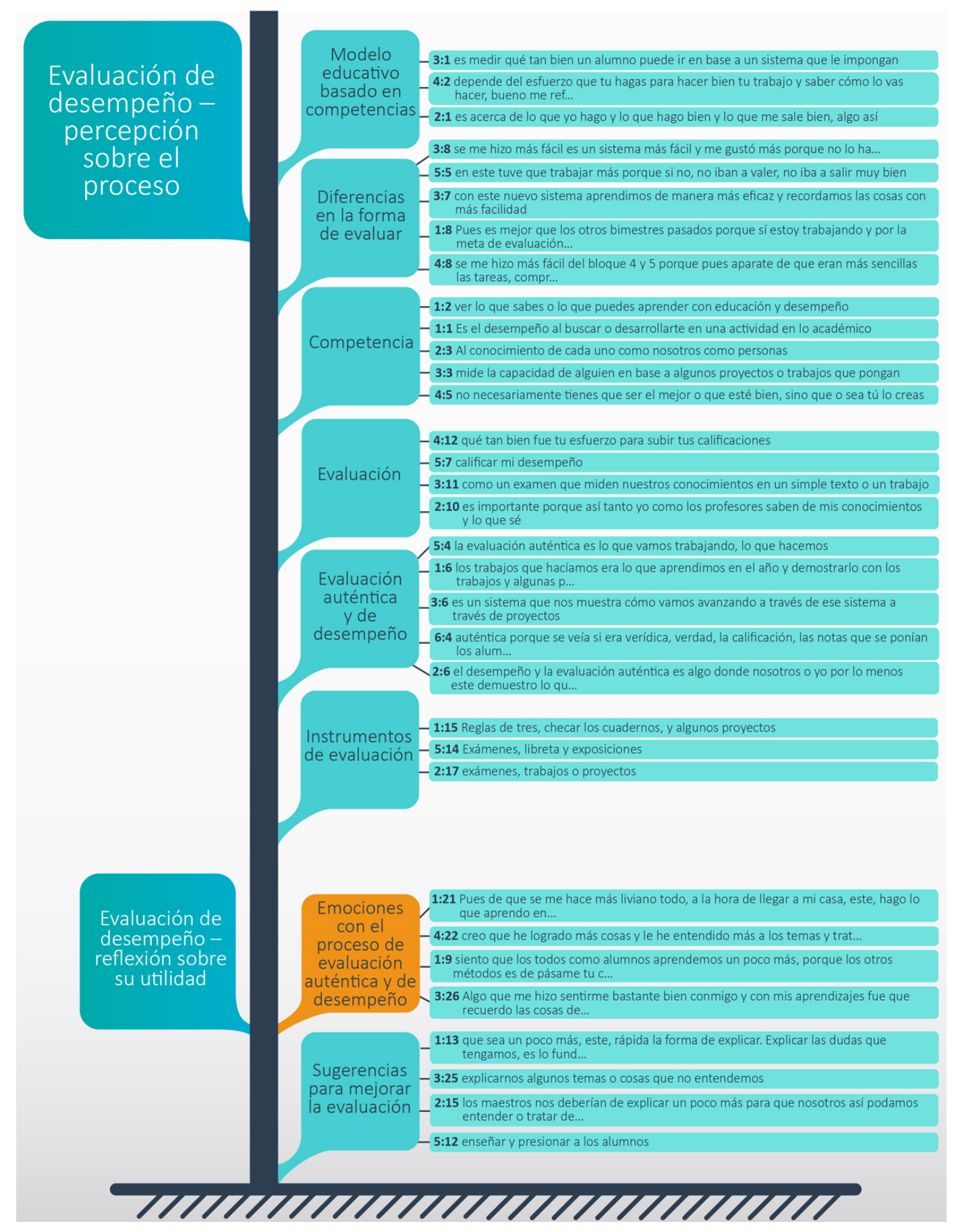

Figura 1. Familia de categorías emergentes sobre evaluación de desempeño: percepción sobre el proceso, analizadas a partir del proceso de indagación sobre la evaluación de desempeño y auténtica

Fuente: Elaboración propia. 


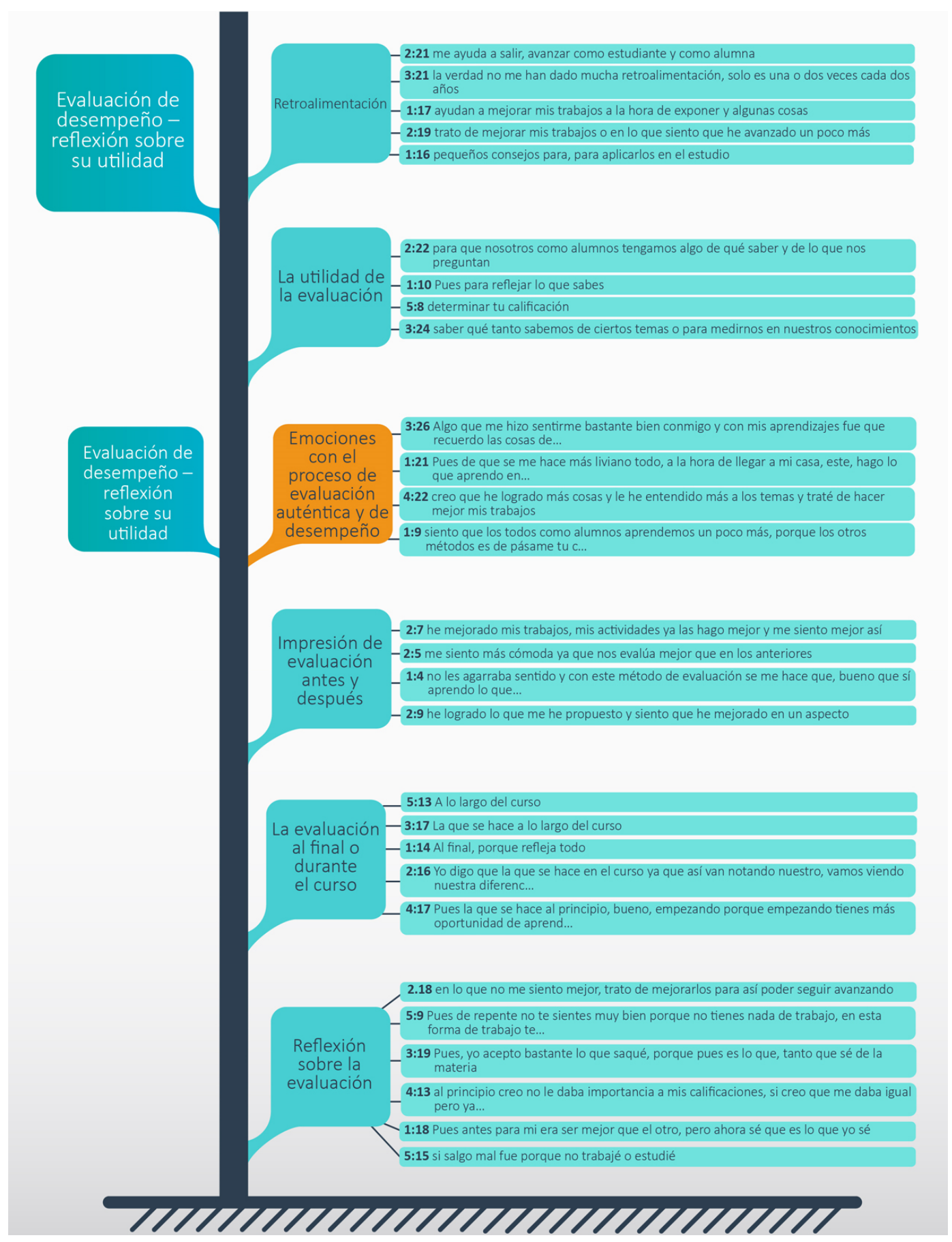

Figura 2. Familia de categorías emergentes sobre evaluación de desempeño: reflexión sobre su utilidad, analizadas a partir del proceso de indagación sobre la evaluación de desempeño y auténtica

Fuente: Elaboración propia.

Además, al opinar sobre diferencias en la forma de evaluar, algunas afirmaciones relevantes fueron:

Con este nuevo sistema aprendimos de manera más eficaz y recordamos las cosas con más facilidad... se me hizo más fácil... es un sistema más fácil. (A 17) 
En este (periodo) tuve que trabajar más porque si no, no iban a valer, no iba a salir muy bien. (A23)

La segunda familia [F2] se formó en torno a lo que piensan los estudiantes que puede ser útil de esta nueva forma de evaluar. Una categoría de esta familia se vincula la percepción que se formó en relación con la evaluación auténtica y del desempeño. Los estudiantes expresaron ideas variadas al respecto:

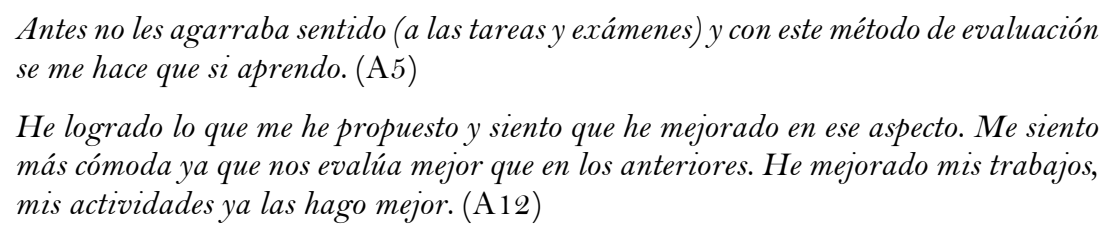

He logrado lo que me he propuesto y siento que he mejorado en ese aspecto. Me siento más cómoda ya que nos evalúa mejor que en los anteriores. He mejorado mis trabajos, mis actividades ya las hago mejor. (A 12)

En cuanto a la retroalimentación, sus respuestas para tratar de definirla, así como hablar sobre su utilidad, reflejan poco contacto con este elemento:

$$
\begin{aligned}
& \text { Son pequeños consejos para aplicarlos en el estudio, ayudan a mejorar mis trabajos a } \\
& \text { la hora de exponer y algunas cosas. (A5) } \\
& \text { La verdad, no me han dado mucha retroalimentación, sólo una o dos veces cada dos } \\
& \text { años. (A17) } \\
& \text { Me ayuda a salir, avanzar como estudiante y como alumna. (A12) }
\end{aligned}
$$

Además, se preguntó si consideraban que sus calificaciones eran mejores en un periodo de tiempo (formativa) o al final (sumativa), en este tema A28 dijo que ambas, a lo largo del curso (A23, A 17 y A 12), un niño prefirió que se hiciera al inicio (A22) y otro que se hiciera al final del curso (A5).

Respecto a lo que piensan sobre sus evaluaciones, sus respuestas fueron: "en lo que no me siento mejor trato de mejorarlo para así poder seguir avanzando" (A 12), "pues de repente no te sientes muy bien porque no tienes nada de trabajo en esta forma de trabajo" (A23), "pues yo acepto bastante lo que saqué porque pues es lo que sé de la materia" (A17), "al principio no le daba importancia a mis calificaciones...pero ahora ya le hecho ganas" (A22), "pues antes para mí era ser mejor que el otro, pero ahora sé que es lo que yo sé" (A5) y "si salgo mal es porque no trabajé o estudié" (A23).

El tema de las emociones lo dejamos al final, abarca las dos familias, sus comentarios, fueron los siguientes: "algo que me hizo sentirme bastante bien conmigo y con mis aprendizajes fue que recuerdo las cosas" (A17), "pues de que se me hace más liviano todo, a la hora de llegar a mi casa hago lo que aprendo en casa" (A5), "creo que he logrado más cosas y le he entendido más a los temas y traté de hacer mejor mis trabajos" (A22) y "siento que todos como alumnos, aprendemos un poco más, porque los otros métodos es de pásame tu cuaderno y copia" (A5).

\subsection{Resultados de tipo cuantitativo}

Se presenta a continuación los resultados del análisis de datos que arrojó la encuesta sobre la evaluación de desempeño y auténtica, en aras de comprender aún mejor la percepción de los estudiantes sobre el cambio en las prácticas de evaluación. La figura 3 muestra los porcentajes obtenidos por pregunta. 


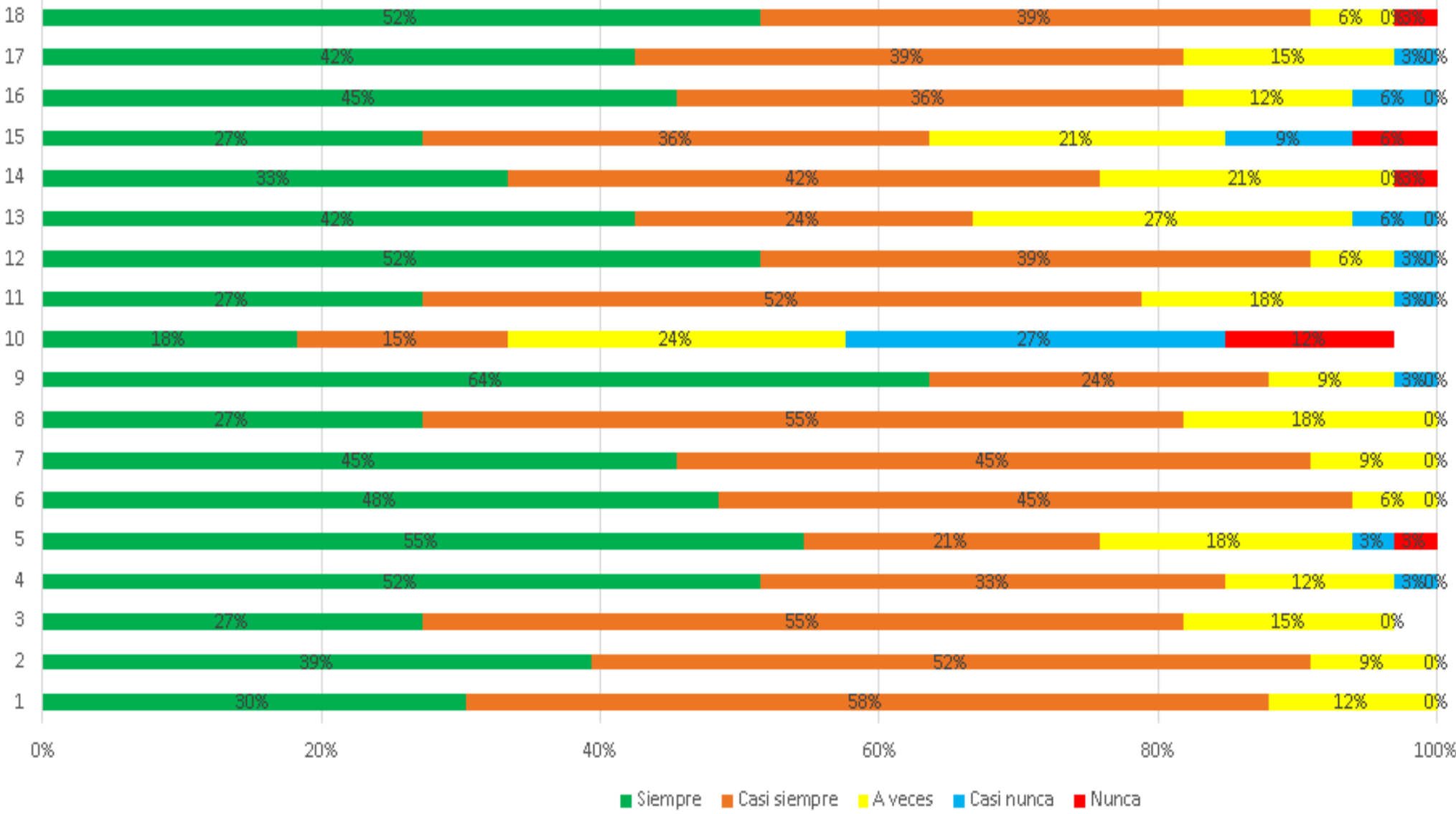

Figura 3. Porcentajes obtenidos en el cuestionario sobre evaluación auténtica y de desempeño Fuente: Elaboración propia. 
Los resultados permiten inferir ciertos aspectos de interés en torno a la percepción de los estudiantes en cuanto a la relación aprendizaje y prácticas de evaluación auténtica y del desempeño.

Con respecto al segmento relacionado con prácticas de evaluación del profesor como son: entrega de resultados y calificaciones, emisión de retroalimentación y modelación para la ejecución de tareas, los estudiantes manifestaron estar de acuerdo en que siempre ( $\mathrm{P}_{1}$ a $\mathrm{P} 5$, entre $30 \%$ y $55 \%$ ) y casi siempre ( $\mathrm{P} 1$ a $\mathrm{P} 5$, del $21 \%$ al $58 \%$ ) el profesor revisa las actividades en forma y tiempo adecuados, da ejemplos para mejorar los trabajos y evalúa de acuerdo con lo preestablecido con ellos.

En relación con el segmento relacionado con el aprendizaje que se suscita a partir de comprender aciertos y errores, la vinculación de lo aprendido con los objetivos formativos, la reflexión que se realiza a partir de resultados para la mejora, los estudiantes coincidieron en su mayoría, en que el profesor abordó los objetivos que se debían alcanzar en cada inicio de bloque y además les indicó cuales eran las mejoras que debían hacer en sus trabajos para mejorar su calificación. Al igual que en caso anterior, los porcentajes de siempre (27\% al $55 \%$ ) y casi siempre $(64 \%$ y $24 \%)$ reflejan un reconocimiento a que esas prácticas se suscitan en el aula.

En cuanto al tercer segmento vinculado, con su experiencia previa con la evaluación de desempeño y auténtica, su aportación al proceso formativo, la relación existente entre aprendizaje y evaluación y la apreciación de una relación positiva entre aprendizaje de la historia y la evaluación de desempeño, en la pregunta 10 respecto a su experiencia previa con este tipo de evaluación, resaltan las respuestas puesto que no solo no tienen conocimiento de este modelo de evaluación sino que no recuerdan haber sido evaluados con algún otro modelo (a veces $24 \%$, casi nunca $27 \%$ y nunca $12 \%$ ); en la pregunta 13 consideraron que esta forma de evaluar les facilitaría el aprendizaje de historia y mejoraría sus calificaciones $(42 \%)$, aunque en la 15 consideraron casi siempre que sus calificaciones se mantuvieron igual con esta forma de evaluar $(36 \%)$.

Finalmente, sobre el segmento (d), la mayoría coincidieron en que esta nueva forma de evaluar mejoraría sus calificaciones en otras materias, les gusta más que otras formas de evaluar y creen que aprenden mejor de esta forma (P $16,45 \%, \mathrm{P} 17,42 \%$ y $\mathrm{P} 18,52 \%)$.

\section{Conclusiones}

Con el fin de superar las críticas respecto al uso de resultados a las evaluaciones tradicionales (diagnóstica, formativa y sumativa), empezaron a surgir en el ámbito educativo nuevas tendencias en evaluación. Por lo menos existen dos más, una de ellas, la evaluación implícita, inserta en el MEBC, que se circunscribe a inferir a través de la observación qué tanto es capaz de realizar el estudiante de las tareas propuestas, cuál es su potencial y lo que hace en realidad para el desarrollo mediante una intervención (Gallardo, 2013).

La otra, es la evaluación auténtica. Este término agrupa todo un conjunto de alternativas frente a la evaluación tradicional, en la evaluación auténtica el alumno hace, crea o produce algo durante un tiempo suficiente que permita evaluar todo el proceso, el resultado o ambos e inclusive el desenvolvimiento del estudiante al momento de estar trabajando (Bravo y Fernández, 2000). 
Este tipo de evaluación ha existido por algunas décadas y se está aplicando en educación superior. Sin embargo, para la educación básica, su puesta en marcha es relativamente nueva. Antes de iniciar esta investigación se indagó acerca de su uso en educación básica y no se halló nada al respecto en revistas o trabajos arbitrados.

En cuanto a las tres familias de análisis que emergieron a partir de la recolección y comprensión del fenómeno de evaluación de desempeño en este estudio, en la primera familia: Evaluación auténtica y del desempeño, se puede inferir que, en general, los jóvenes desconocen lo que son las competencias, a pesar de que vienen señaladas en los planes y programas. No es algo que los docentes platiquen o planteen a sus alumnos, tampoco tienen claro lo que es una evaluación, tienden a confundirla con calificación (Fernández, 2010, Gallardo, 2013, Córdova, 2010). Se pudo inferir también que los estudiantes, si son tratados como personas responsables, responden como tales. En las entrevistas, todos los alumnos fueron consistentes en su respuesta y más cercanos a lo que se les explicó que era, es decir, respondieron en su papel de estudiantes, consideraron a la evaluación que se les hizo como más amigable y accesible, dado que sabían cómo serían evaluados durante todo el proceso y lo que se esperaba de ellos.

La segunda familia denominada: Evaluación auténtica y del desempeño -reflexión sobre su utilidad-, aportó información valiosa respecto a lo que piensan los estudiantes que puede ser útil de esta nueva forma de evaluar. Los estudiantes compararon lo que fue esta evaluación con las que han tenido antes o que tuvieron en otras materias e incluso con la evaluación de los primeros tres bloques de historia durante el ciclo escolar 2016-2017, sus respuestas fueron que ahora sí tenía sentido su evaluación, era mejor que otras, mejoraron su calificación y la calidad de sus trabajos.

A pesar de ser opiniones de niños y niñas, de diferentes promedios y actitudes hacia el trabajo escolar, todos estuvieron abiertos a esta nueva forma de evaluar, aceptaron de buen grado el cambio en la evaluación y manifestaron estar de acuerdo (comentaron que le entendían más y era fácil), en que se les evaluará así en la materia e incluso en las demás; coincidieron que deben aceptar retroalimentaciones para mejorar, que una forma de evaluarlos es demostrar lo que saben en cualquier momento y entienden que las competencias no se refieren precisamente a una justa deportiva (Alonso, Gil y Martínez, 1996; Fernández March, 2010; Gallardo, 2013; Hattie, 2011). El término de retroalimentación es desconocido para ellos, a pesar de existir en los planes y programas, al igual que las competencias, casi no se llevan a cabo por la carga de trabajo que da atender diversos y numerosos grupos.

Además de lo recolectado, en cuanto a experiencia alrededor de la evaluación del desempeño, es inevitable registrar ciertas emociones que emergen durante el proceso de investigación. En ese sentido, aunque la emoción es algo difícil de describir, en la presente investigación se registraron gestos y expresiones de satisfacción en la entrevista al hablar de la evaluación auténtica y del desempeño, ellos expresaron sentirse a gusto y satisfechos con esta forma de evaluar, consideraron que es mejor que otras formas de evaluar y los hace rendir más y mejor

Estuvieron de acuerdo en que ellos, como alumnos, tienen que estar conscientes de lo que van a aprender, los aspectos a mejorar en cada actividad posterior gracias a las retroalimentaciones que les da su profesor. Todos dijeron desconocer la evaluación auténtica y del desempeño, pero coincidieron en que entendieron de qué se trataba, les gusto y creen que mejorarán sus calificaciones si se aplica esta evaluación en otras 
materias. También coincidieron en que gracias a esta forma de evaluar sus calificaciones subieron en la materia de historia o en su defecto aprendieron más y mejor que con otras formas de evaluación que han tenido.

La evaluación auténtica y del desempeño mostró su valía en este proyecto, pero para dar por sentado que es un proceso útil en este nivel educativo, se tendría que aplicar e implementar a varios grupos en diversas materias, algo un poco difícil pues no existe una capacitación en cuanto a su utilización en este medio.

La investigación muestra cómo los estudiantes interactúan cotidianamente con el conocimiento y no son meros recipientes que se deben llenar de procedimientos y qué si se es buen guía, se podrá hacer de ellos jóvenes más competentes en todas las áreas que decidan incursionar. Esta investigación puede servir como referencia (de forma modesta) para futuras investigaciones en este nivel educativo, a pesar de los planes y programas que se han desarrollado, poco o casi nada hay desarrollado sobre la aplicación y resultados obtenidos de las diversas formas de evaluación en educación secundaria y los procesos de enseñanza-aprendizaje.

Las limitaciones de este estudio se relacionan con el hecho de haberlo realizado en un solo escenario educativo. Los futuros estudios en esta misma línea de investigación podrían diseñarse para indagar más sobre: (a) el acercamiento de los estudiantes a alcanzar el nivel que señalan las competencias de egreso con apoyo de la retroalimentación y (b) entender cómo los diferentes mecanismos de evaluación de desempeño y auténtica inciden en la apropiación de conocimiento y desarrollo de competencias disciplinares y transversales a partir del estudio de la Historia como disciplina escolar.

\section{Referencias}

Aguerrondo, I. (2008). Conocimiento complejo y competencias educativas. Recuperado a partir de http://www.ibe.unesco.org/sites/default/files/resources/wpci-08knowledge_compet_spa.pdf

Alonso, M., Gil, D. y Martínez, J. (1996). Evaluar no es calificar. La evaluación y la calificación en una enseñanza constructivista de las ciencias. Investigación en la Escuela, 30, 15-26.

Argudín, Y. (2010). Educación basada en competencias. Nociones y antecedentes. Ciudad de México: Trillas.

Bravo, A. y Fernández, J. (2000). La evaluación convencional frente a los nuevos modelos de evaluación auténtica. Psicothema, Revista Anual de Psicología, 12(2), 95-99.

Carretero, M. (2000). Cambio conceptual y enseñanza de la historia. Tarbiya, 26, 73-82.

Casal, S. (2011). Aprender historia en la escuela secundaria. El caso de Morelia, Michoacán (México). Revista Mexicana de Investigación Educativa, 16(48), 73-105.

Chagoyan, P. (2013). Rastreando el origen pedagógico del modelo educativo por competencias. Recuperado de http://www.revistacoepesgto.mx/revistacoepes7/index.php/rastreandoel-origen-pedagogico-del-modelo-educativo-por-competencias

Chong, M. A. (2013). Sistema educativo en México: El modelo de competencias, de la industria a la educación. Sincronía, 63, 1-6.

Córdova, A. M. (2010, abril). Evaluación de la educación. Comunicación presentada en Congreso Iberoamericano de la Educación. Buenos Aires, Argentina. 
Creswell, J. y Plano, V. (2007). Designing and conducting mixed methods research. Thousand Oaks, CA: SAGE.

De Ketele, J. M. (2008). Enfoque socio-histórico de las competencias en la enseñanza. Profesorado, Revista de Currículum y Formación del Profesorado, 12(3), 1-12.

De la Orden Hoz, A. (2011). Reflexiones en torno a las competencias como objeto de evaluación en el ámbito educativo. Revista Electrónica de Investigación Educativa, 13(2), 1-21.

Escamilla, J., Quintero, E., Fuerte, K., Venegas, E., Fernández, K., Elizondo, J. y Román, R. (2016). Evaluación del desempeño en el modelo educativo basado en competencias. Observatorio de Innovación Educativa. Edu Trends, 36, 1-23.

Fernández, A. (2010). La evaluación orientada al aprendizaje en un modelo de formación por competencias en la educación universitaria. Revista de Docencia Universitaria, 8(1), 11-34. https://doi.org/10.4995/redu.2010.6216

Ferrer, G. (2006). Sistemas de evaluación de aprendizajes en América Latina. Balance y desafios. Buenos Aires: PREAL.

Flores-Kastanis, E. y De la Torre Gamboa, M. (2010). La problemática de la investigación sobre cambio educativo. Revista Mexicana de Investigación Educativa, 15(47), 1017-1023.

Fullan, M. (2002). El significado del cambio educativo: Un cuarto de siglo de aprendizaje. Profesorado, Revista de Currículum y Formación del Profesorado, 6(1), art 1.

Gallardo, K. E. (2013). Evaluación del aprendizaje: Retos y mejores prácticas. Monterrey: Tecnológico de Monterrey.

Gallardo, K. E., Alvarado García, M. A., Lozano Rodríguez, A., López Cruz, C. S. y Gudiño Paredes, S. (2017). Materiales digitales para fortalecer el aprendizaje disciplinar en educación media superior: Un estudio para comprender cómo se suscita el cambio educativo. REICE. Revista Iberoamericana sobre Calidad, Eficacia y Cambio en Educación, 15(2), 89-109. https://doi.org/10.15366/reice2017.15.2.005

Ginés, J. (2004). La necesidad del cambio educativo para la sociedad del conocimiento. Revista Iberoamericana de Educación, 35, 13-37.

Hidalgo, N. y Murillo, F. J. (2017). Las concepciones sobre el proceso de evaluación del aprendizaje de los estudiantes. REICE. Revista Iberoamericana sobre Calidad, Eficacia y Cambio en Educación, 15(1), 107-128. https://doi.org/10.15366/reice2017.15.1.007

Hopenhayn, M. (2003). Educación, comunicación y cultura en la sociedad de la información: Una perspectiva latinoamericana. Santiago de Chile: Naciones Unidas.

López, M. I. (2011). Evaluación auténtica de los aprendizajes en Historia y Ciencias Sociales, problemática y alcances metodológicos. Horizontes Educacionales, 16(2), 73-92.

Monereo, C. (2009). La autenticidad de la evaluación. En C. Castelló (Coord.), La evaluación auténtica en enseñanza secundaria y universitaria (pp. 312-354). Barcelona: Edebé.

Murillo, F. J. y Román, M. (2008). Resultados de aprendizaje en América Latina a partir de las evaluaciones nacionales. Revista Iberoamericana de Evaluación Educativa, 1(1), 6-35.

OEI. (2010). Metas educativas 2021. Recuperado de http://www.oei.es/historico/metas2021/libro.htm

Rodríguez, I. y Gallardo, K. E. (2017). Redesigning an educational technology course under a competency-based performance assessment model. Pedagogika, 127(3), 184-204. https://doi.org/10.15823/p.2017.48

SEP. (2011). Plan de estudios 2011. Ciudad de México: SEP. 
SEP. (2013a). Acuerdo 696 por el que se establecen normas generales para la evaluación, acreditación, promoción y certificación en la educación básica. Ciudad de México: SEP.

Tobón, S. (2008). La formación basada en competencias en la educación superior: El enfoque complejo. Ciudad de México: SEP.

Tójar, J. C. (2006). Investigación cualitativa: Comprender y actuar. Madrid: La Muralla.

Wolfe, R., Valverde, G. y Esquivel, J. M. (2000). Los próximos pasos: ¿Hacia dónde y cómo avanzar en la evaluación de aprendizajes en América Latina? Buenos Aires: PREAL.

\section{Apéndice A}

Cuadro 1. Entrevista a profundidad

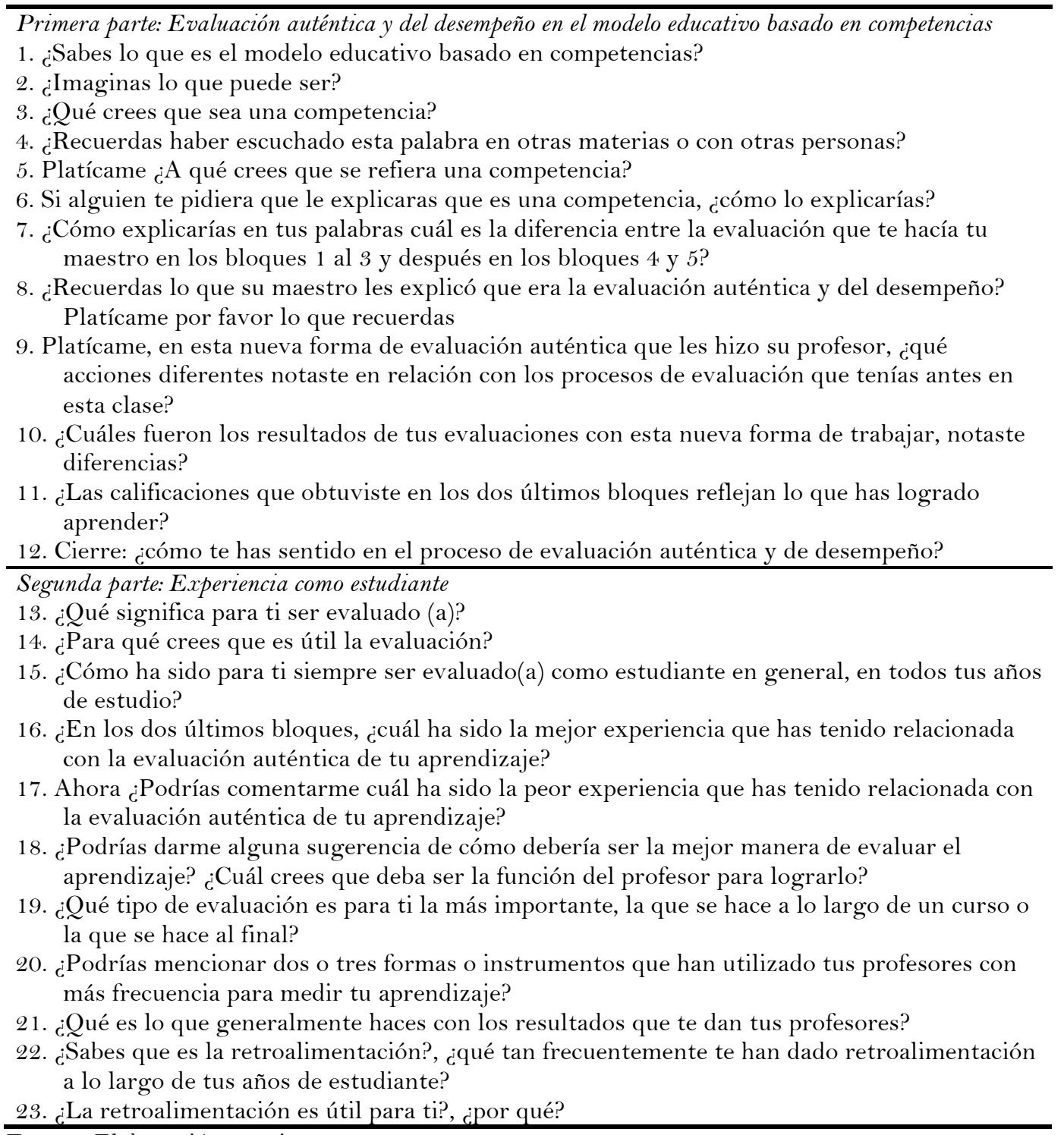

Fuente: Elaboración propia. 


\section{Apéndice B}

\section{Encuesta sobre percepción de la evaluación auténtica y del desempeño}

Este instrumento busca recolectar la opinión de los estudiantes de $2^{\circ}$ grado, grupo G, en relación con la evaluación auténtica y del desempeño que reciben sobre sus trabajos académicos, actividades y ejercicios en general del $4^{\circ}$ y $5^{\circ}$ bloque del ciclo escolar 20172018

\section{Nombre del alumno:}

Prof. Aliosha Alderete Martínez.

Materia: Historia I

Escuela Secundaria Técnica 116 "Nezahualpilli"

Instrucciones: Contesta a las siguientes afirmaciones calificando cada una de las ideas. Marca con una $\mathbf{X}$ el número que mejor califique cada afirmación según tu opinión. Usa la siguiente escala: 1 Totalmente de acuerdo, 2 De acuerdo, 3 Imparcial, 4 En desacuerdo, 5 Totalmente en desacuerdo.

\begin{tabular}{|c|c|c|c|c|c|}
\hline & 1 & 2 & 3 & 4 & 5 \\
\hline Mi profesor califica (evalúa) mis trabajos frecuentemente. & & & & & \\
\hline Mi profesor me entrega calificaciones y comentarios a mis trabajos sin demora. & & & & & \\
\hline Mi profesor me solicita que yo investigue en diversos medios sobre mis tareas. & & & & & \\
\hline Mi profesor me pone ejemplos sobre cómo hacer mejor mis tareas. & & & & & \\
\hline $\begin{array}{l}\text { La calificación (evaluación) hecha por mi profesor sobre mis tareas me ayuda a } \\
\text { aprender mejor. }\end{array}$ & & & & & \\
\hline
\end{tabular}

\section{Responde a partir de la última vez que tu profesor(a) te encargo un trabajo}

\begin{tabular}{|c|c|c|c|c|c|}
\hline & 1 & 2 & 3 & 4 & $\mathbf{5}$ \\
\hline $\begin{array}{l}\text { Mi profesor me dijo cuál era el objetivo de aprendizaje de los trabajos que } \\
\text { íbamos a realizar. }\end{array}$ & & & & & \\
\hline $\begin{array}{l}\text { Después de mi calificación entendí qué tan cerca o lejos estoy de lograr lo que } \\
\text { el maestro nos dijo que debíamos aprender. }\end{array}$ & & & & & \\
\hline $\begin{array}{l}\text { Ahora sé qué debo cuidar o mejorar en los siguientes trabajos para que me vaya } \\
\text { mucho mejor y logre aprender lo que el maestro nos dijo. }\end{array}$ & & & & & \\
\hline $\begin{array}{l}\text { Cuando mi profesor evaluó mi trabajo, me hizo comentarios sobre qué tan bien } \\
\text { o mal lo hice. }\end{array}$ & & & & & \\
\hline Esta forma de trabajo yo ya la había tenido antes con otros profesores & & & & & \\
\hline Entiendo lo que es la evaluación del desempeño y auténtica & & & & & \\
\hline $\begin{array}{l}\text { Esta forma de trabajo me gusta más que la forma en que el maestro nos había } \\
\text { estado evaluando de los bloques } 1 \text { al } 3 \text {. }\end{array}$ & & & & & \\
\hline Creo que aprendo mejor con esta forma de evaluación. & & & & & \\
\hline $\begin{array}{l}\text { Sé que si aprendo hacer las actividades y las puedo explicar mi calificación será } \\
\text { mejor }\end{array}$ & & & & & \\
\hline Mi calificación subió en esta materia con esta nueva forma de evaluar & & & & & \\
\hline $\begin{array}{l}\text { Tal vez mis calificaciones de otras materias subirían con esta nueva forma de } \\
\text { evaluar }\end{array}$ & & & & & \\
\hline $\begin{array}{l}\text { Mi calificación en esta materia se mantuvo igual con esta nueva forma de } \\
\text { evaluar. }\end{array}$ & & & & & \\
\hline Me gusta esta nueva forma de evaluación auténtica y de desempeño. & & & & & \\
\hline
\end{tabular}




\section{Breve CV de los autores}

\section{Aliosha Alderete Martínez}

Docente en la Escuela Secundaria Técnica 116 "Nezahualpilli" del Estado de México, donde imparte las materias de Historia y Formación Cívica y Ética. Tiene estudios de licenciatura en Sociología por la Universidad Nacional Autónoma de México (UNAM), es diplomado en Derechos humanos y sistema penal (UNAM) y tiene una maestría en Educación por el Tecnológico de Monterrey (ITESM). Ha sido bailarín y maestro de bailes regionales durante más de 10 años; está interesado en el mantenimiento y divulgación de los bailes populares de México, la enseñanza de las ciencias sociales a nivel básico y el proceso de enseñanza aprendizaje dentro del Modelo Educativo Basado en Competencias, tiene intención en promover la evaluación auténtica y de desempeño en el marco del cambio educativo. ORCID ID: https://orcid.org/0000-0003-2920-8625. Email: almaragon@gmail.com

\section{Katherina Edith Gallardo}

Docente e investigadora de la Escuela de Humanidades y Educación del Tecnológico de Monterrey. Es miembro del Sistema Nacional de Investigadores avalado por el CONACyT (Nivel 1). Tiene estudios de licenciatura en Pedagogía por el Instituto Pedagógico Nacional de Monterrico (IPNM, Lima - Perú), de maestría en Psicología Educativa por la Universidad Nacional Autónoma de México (UNAM) y de doctorado en Innovación y Tecnología Educativa por el Tecnológico de Monterrey (ITESM). Coordina un grupo de asesores tutores de tesis de maestría y ha dirigido varias disertaciones doctorales. Sus líneas de investigación giran alrededor de los temas relacionados con evaluación del aprendizaje, modelo educativo basado en competencias y educación a distancia, entre las principales. ORCID ID: https://orcid.org/0000-0001-8343-9518. Email: katherina.gallardo@itesm.mx 